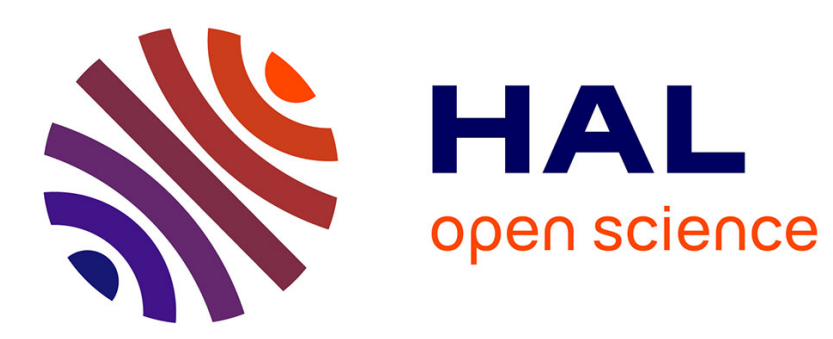

\title{
The Subsurface Diffusion of Hydrogen on Rutile TiO2 Surfaces: A Periodic DFT Study
}

Baohuan Wei, Monica Calatayud

\section{To cite this version:}

Baohuan Wei, Monica Calatayud. The Subsurface Diffusion of Hydrogen on Rutile TiO2 Surfaces: A Periodic DFT Study. Topics in Catalysis, 2021, 10.1007/s11244-021-01518-w . hal-03419562

\section{HAL Id: hal-03419562 \\ https://hal.science/hal-03419562}

Submitted on 8 Nov 2021

HAL is a multi-disciplinary open access archive for the deposit and dissemination of scientific research documents, whether they are published or not. The documents may come from teaching and research institutions in France or abroad, or from public or private research centers.
L'archive ouverte pluridisciplinaire HAL, est destinée au dépôt et à la diffusion de documents scientifiques de niveau recherche, publiés ou non, émanant des établissements d'enseignement et de recherche français ou étrangers, des laboratoires publics ou privés. 


\section{The subsurface diffusion of hydrogen on rutile $\mathrm{TiO}_{2}$ surfaces: A periodic DFT study}

\section{Baohuan Wei and Monica Calatayud*}

Laboratoire de Chimie Théorique, CNRS, Sorbonne Université, 4 Place Jussieu, Paris, 75005, France.

* Laboratoire de Chimie Théorique, CNRS, Sorbonne Université,

4 Place Jussieu, Paris, 75005, France.

E-mail(s): calatayu@lct.jussieu.fr;

Contributing authors: baohuanw@1ct.jussieu.fr;

\section{Abstract}

Titanium oxide $\left(\mathrm{TiO}_{2}\right)$ is an oxide with a wide range of technological and industrial applications. The interaction of hydrogen with $\mathrm{TiO}_{2}$ surface is an important step in many catalytic processes, such as spillover or water photosplitting. In the last years, the role of the subsurface hydrogen has been invoked many times to explain different phenomena, from black titania to reversible storage. In this work, we systematically investigate the paths for hydrogen diffusion from surface into subsurface focusing on the surface topology, the thermal and isotopic effects, and the degree of reduction of the substrate, by means of state of the art periodic DFT calculations. We find differences in the behavior of the rutile $\mathrm{TiO}_{2}$ surfaces (001), (100) and (110) slightly exothermic reaction energies from $-0.02 \mathrm{eV}$ to $-0.15 \mathrm{eV}$, and activation energies from $0.39 \mathrm{eV}$ to $1.00 \mathrm{eV}$. Deuterium diffusion from rutile (110) surface to the bulk, as well as thermal effects, were found to affect less than $0.04 \mathrm{eV}$ the energy profile. On the contrary, the degree of reduction of the rutile (110) was found to noticeably decrease the activation barrier down to $0.8 \mathrm{eV}$ for the (110) slab. We analyze structural, electronic and reactivity parameters that affect the kinetic barriers for the surface to subsurface $\mathrm{H}$ diffusion, and conclude that topology and reduction degree are valid strategies to tune the surface-tobulk migration process.

Keywords: hydrogen diffusion, topology effect, rutile $\mathrm{TiO}_{2}$ isotopic effect, thermal correction 


\section{Introduction}

$\mathrm{TiO}_{2}$ has been widely studied as a model metal oxide with a large number of applications, such as solar cells [1, 2], sensors [3, 4], photocatalysts [5] or heterogeneous catalysts [6]. The interaction of hydrogen with $\mathrm{TiO}_{2}$ surfaces is an important step in many reaction processes, such as hydrogen storage, hydrogen spillover or photocatalytic water splitting to cite a few. In the last years many studies have pointed out the importance of subsurface hydrogen, which could be determining for many observations such as the reversible absorption in anatase nanoparticles [7], the stabilization of surface hydrogen intermediates [8], the properties of black titania [9], its role in hydrogen permeable membranes [10], or the infrared baseline of titania-based catalysts [11]. In the present work we investigate the mechanisms for subsurface diffusion in rutile substrates, focusing on the role of the topology, the degree of reduction and the isotopic effect, by means of state of the art theoretical tools based on density functional theory (DFT).

Frequently, adsorbed hydrogen is observed on $\mathrm{TiO}_{2}$ surfaces due to the standard surface science cleaning procedures [12], or is achieved by direct adsorption of atomic atoms on single crystals [13], and photolysis of adsorbed carboxylic acids [14]. The activity of the $\mathrm{TiO}_{2}$ is highly affected by extrinsic (hydrogen impurity) defects, which supply a charge excess. As a consequence, the electronic properties as well as the bandgap of $\mathrm{TiO}_{2}$ can be influenced [15, 16]. Moreover, many reports have shown that the hydrogenation of $\mathrm{TiO}_{2}$ can boost the photo-absorption of $\mathrm{TiO}_{2}$ and its photocatalytic activity for the splitting of water to $\mathrm{H}_{2}$ [17-22]. Numerous experimental and theoretical works have been carried out to study the $\mathrm{H}-\mathrm{TiO}_{2}$ system [10, 12, 23-28]. It was widely proved that atomic $\mathrm{H}$ instead of molecular $\mathrm{H}_{2}$ can react with $\mathrm{TiO}_{2}$ surface. Still, a clear picture of the physico-chemical features affecting $\mathrm{H}$ migration to the subsurface is still missing.

In 2008, some of us reported for the first time indirect evidence for the stabilization of bulk hydrogen in a $\mathrm{TiO}_{2}$ single-crystal [13], which were consistent with the experimental observations: heating of the hydrogenated sample removes surface hydrogen but does not lead to recombinative desorption of $\mathrm{H}_{2}\left(\right.$ or $\mathrm{H}_{2} \mathrm{O}$ ) molecules, with a barrier of $1.11 \mathrm{eV}$. The presence of stable subsurface hydrogen was confirmed experimentally by an 
independent group one year later [29]. Subsurface hydrogen atoms are found to reside in a stable interstitial site as subsurface $\mathrm{OH}$ groups detectable in scanning tunneling microscopy. Some years later we predicted that the migration to the bulk could also occur in anatase $\mathrm{TiO}_{2}(101)$, requiring barriers varying from 0.70 to $2.25 \mathrm{eV}$, depending on the diffusion path [26].

Selloni et al. reported the reversible incorporation into cubic and octahedral $\mathrm{TiO}_{2}$ anatase nanocrystals [7], and later provided valuable experimental and theoretical information on different coverages and pathways, possible adsorption sites, and the presence of defects for $\mathrm{H}$ diffusion on anatase (101) [25]. Recently, a combined theoretical and experimental study confirmed the subsurface diffusion of $\mathrm{H}$ atom in anatase $\mathrm{TiO}_{2}$ by observing a hydrogenated surface shell, which is formed through subsurface diffusion of photo-reduced water protons generated at the aqueous $\mathrm{TiO}_{2}$ interface. An overall theoretical barrier for the preferred pathway of $0.77 \mathrm{eV}$ [30] was determined.

The diffusion of $\mathrm{H}$ in $\mathrm{TiO}_{2}$ including anatase and rutile $\mathrm{TiO}_{2}$ has been studied by various experimental methods [31,32]. Ohashi et al. investigated the hydrogenation of rutile $\mathrm{TiO}_{2}(110)$ surface by low-energy hydrogen ions, by means of ultraviolet photoemission spectroscopy (UPS), X-ray photoemission spectroscopy (XPS) and nuclear reaction analysis (NRA) [31]. It was found that $\mathrm{H}$ atoms are distributed to a depth of $30 \mathrm{~nm}$ with an average concentration of 5.6 at.\%. Subsurface sites were confirmed to be stable sites for hydrogen. Moreover, N. Nagatsuka et al. carried out similar experiment, but for anatase (101) at different temperature (200K and 300K) [32]. Their results showed that $\mathrm{H}$ diffusion in anatase $\mathrm{TiO}_{2}$ is much faster than in rutile $\mathrm{TiO}_{2}$.

It is well known that the surface properties strongly vary with different crystallographic orientations, which can greatly affect their reactivity [33, 34]. While previous studies mainly focus on one surface, there is no systematic investigation with various orientations for $\mathrm{H}$ diffusion on $\mathrm{TiO}_{2}$. In this work we address the role of the termination in the diffusion mechanisms of hydrogen for three terminations of rutile $\mathrm{TiO}_{2}$. We furthermore analyze how geometry and electronic structure affect the migration energetic profile.

Other phenomena could also modify the diffusion of hydrogen to the 
lattice. For instance, quantum tunneling due to the light weight of hydrogen could facilitate migration [35]. Thermal effects may also increase the migration rate. Besides, the presence of excess electrons due to the easy reduction of $\mathrm{Ti}^{4+}$ to $\mathrm{Ti}^{3+}$ might also have an impact in the reaction barriers, as shown recently for water adsorption/desorption processes [36]. In order to address these effects, we include in the present work isotopic substitution by deuterium, thermal corrections and substrate degree of reduction for the (110) termination. We discuss the results and suggest that the morphology of the surface and the degree of reduction of the substrate should play key roles in the control of $\mathrm{H}$ surface to bulk migration phenomena.

\section{Computational methods}

Periodic DFT calculations have been performed in the generalized gradient approximation and the Perdew-Berke Ernzerhof functional PBE [37] as implemented in the Vienna ab initio simulation package (VASP) [38, 39] 5.4.4 version. Projector Augmented Wave (PAW) pseudopotentials were applied to describe the core electron representation, with 1, 4 and 6 valence electrons for $\mathrm{H}$, Ti and $\mathrm{O}$ respectively $[38,39]$. The GGA+U approach of Dudarev et al was used to treat the $3 \mathrm{~d}$ electrons of Ti with the effective Hubbard on-site Coulomb interaction parameter (U' = U J) [40]. We choose U' $=4$ according to the proposed value from previous works [26, 41]. A $400 \mathrm{eV}$ cutoff energy for the plane-wave basis set was found to be sufficient in the case of rutile surface [13]. The energies were converged to $10^{-4} \mathrm{eV}$ for the electronic loop, and the forces acting on each atom were smaller than $0.03 \mathrm{eV} / \AA$.

The adsorption energy, or binding energy $\left(\mathrm{E}_{b}\right)$ of the hydrogen on oxygen sites is calculated according to the equation below:

$$
E_{b}=E_{\text {slab/H }}-E_{\text {slab }}-\frac{1}{2} E_{H_{2}}
$$

where $\mathrm{E}_{s l a b}$ and $\mathrm{E}_{s l a b / H}$ indicate the energies of the bare slab and with hydrogen adsorbed on the slab, respectively, and $\mathrm{E}_{\mathrm{H}_{2}}$ is the energy of a hydrogen molecule in a box $(-6.76 \mathrm{eV})$.

In this study, (001), (100), and (110) terminations of rutile $\mathrm{TiO}_{2}$ 
were investigated. The size, composition, coordination number of surface sites for three slab models are shown in Fig. 1 and Table 1. These three stoichiometric facets were modeled as a two-dimensional slab in a three-dimensional periodic cell. The slabs were cleaved from relaxed rutile $\mathrm{TiO}_{2}$ bulk unit cell. For the part of hydrogen migration in bulk, a $2 \times 2 \times 2$ bulk supercell is used for comparison with slabs. All our slabs consist of eight $\mathrm{TiO}_{2}$ units, and were built with the MAPS software [42], with eight $\mathrm{TiO}_{2}$ layers in thickness for (001), (100) and four $\mathrm{TiO}_{2}$ layers for (110). A vacuum thickness of $20 \AA$ was introduced to prevent interaction between slabs. The bottom-half layers of the slab models were kept frozen and the upper-half layers were allowed to relax.

As regards the coverage, a range of values is reported in the literature for (110): $0.25 \mathrm{ML}$ is found in ref. [43], 0.66 ML is found after exposure to high amounts of $\mathrm{H}$ in ref. [13], and $1 \mathrm{ML}(1 \times 1$ pattern) is characterized in ref. [44]. Based on the latter, we have chosen to model high coverage for all the terminations considered. In order to analyze the effect of the coverage, we have computed the adsorption energy in bigger unit cells (See SI Table S 1) and the results are discussed below.

The CI-NEB methods (constrained minimization and climbingimage nudged elastic band methods) were used for searching TS (transition states) [45]: the minimum energy pathway for each elementary reaction was discretized by a total of 4 images between the initial and final states. Then the Improved Dimer Method (IDIM) was applied to find the stationary point [46]. All the transition states were identified by performing the frequency analysis. Only one imaginary frequency was obtained and was checked to connect to the initial and final states. The structures for initial, TS and final systems for the three slabs can be found in the supporting information. The zero point energy (ZPE) was computed as one-half of the sum of real vibration frequencies, to correct adsorption energies. 
Table 1 Size, composition, layers, coordination numbers for three rutile $\mathrm{TiO}_{2}$ surfaces.

\begin{tabular}{llll}
\hline & $(001)$ & $(100)$ & $(101)$ \\
Supercell & $1 \times 1$ & $1 \times 1$ & $1 \times 1$ \\
Composition $\left(\mathrm{TiO}_{2}\right.$ units $)$ & 8 & 8 & 8 \\
$\mathrm{TiO}_{2}$ layers (frozen/relaxed) & $4 / 4$ & $4 / 4$ & $2 / 2$ \\
Atoms (frozen/relaxed) & $12 / 12$ & $12 / 12$ & $12 / 12$ \\
Coordination & $\mathrm{Ti}(4), \mathrm{O}(2,3)$ & $\mathrm{Ti}(5), \mathrm{O}(2,3)$ & $\mathrm{Ti}(5,6), \mathrm{O}(2,3)$ \\
Parameter $(\mathrm{a} . \mathrm{b})(\AA)$ & $\mathrm{a}=\mathrm{b}=4.661$ & $\mathrm{a}=4.661, \mathrm{~b}=2.962$ & $\mathrm{a}=2.962, \mathrm{~b}=6.592$ \\
\hline
\end{tabular}

The Gibbs free energy at room temperature (298K) was also considered within the following approximations:

$G(T)=H-T S=U+p V-T S \approx U(T)-T S(T)$

For the adsorption system, it is reasonable to consider only the vibrational contributions:

$U(T)=E_{D F T}+E_{Z P E}+U_{v i b}(T)$

$S(T)=S_{\text {vib }}(T)$

$U_{v i b}(T)=\sum_{i=1}^{3 N-6}\left(\frac{h v_{i}}{e^{-\frac{h v_{i}}{K_{B} T}-1}}\right)$

$S_{v i b}(T)=K_{B} \sum_{i=1}^{3 N-6}\left(\frac{h v_{i}}{K_{B} T\left(e^{-\frac{h v_{i}}{K_{B} T}-1}-1\right)}\right)-\ln \left(1-\frac{h v_{i}}{K_{B} T}\right)$

$G(T) \approx F(T)=E_{D F T}+E_{Z P E}+K_{B} T \sum_{i=1}^{3 N-6}\left[\ln \left(1-e^{-\frac{h v_{i}}{K_{B} T}}\right)\right]$

where $\mathrm{G}$ is the Gibbs free energy of the system for temperature $\mathrm{T}$ and pressure $\mathrm{P}, \mathrm{S}$ is entropy, $\mathrm{H}$ is enthalpy and $\mathrm{U}$ is internal energy. 


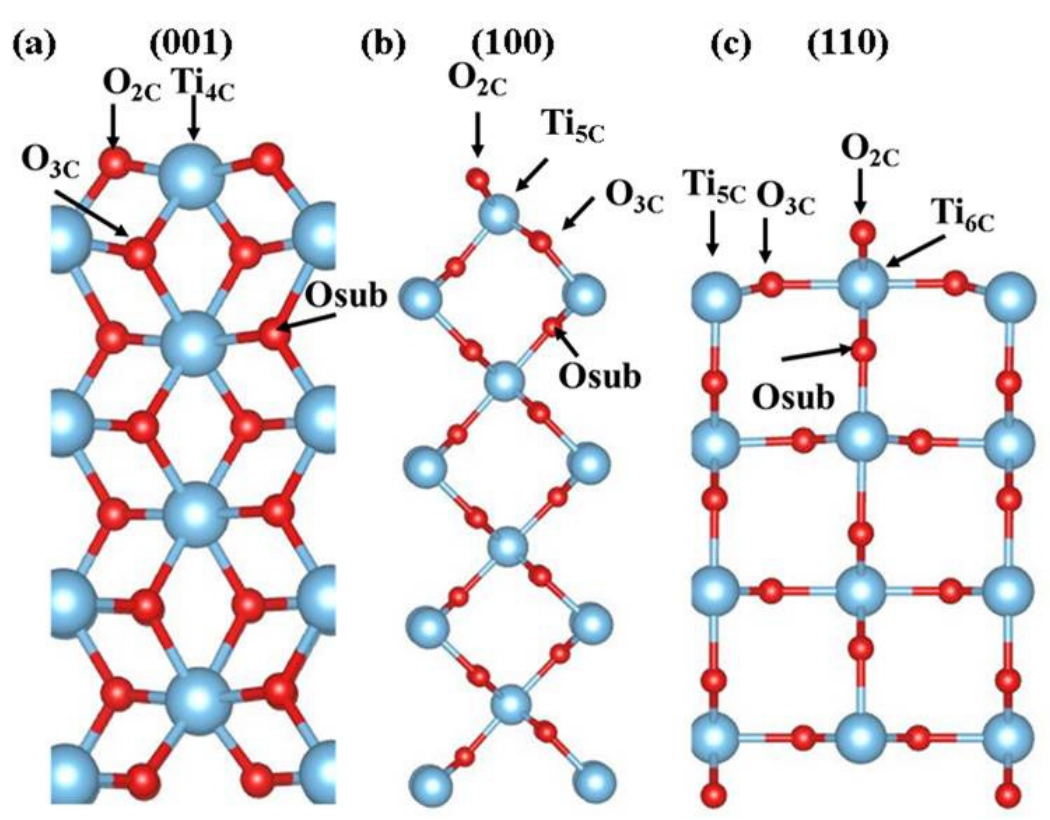

Fig. 1 Side views of rutile $\mathrm{TiO}_{2}$ (001) (a), (100)(b), (110) (c) surfaces. Ti, blue; O, red.

\section{Results and discussion}

3.1 Adsorption of $\mathrm{H}$ on rutile $\mathrm{TiO}_{2}$ surfaces (001), (100),

(110)

The adsorption of one $\mathrm{H}$ was studied by placing the $\mathrm{H}$ atom at different sites of surface and subsurface for rutile (001), (100) and (110) surface in a 1 $\mathrm{x} 1$ unit cell. The oxygen sites considered are labeled as $\mathrm{O}_{2 C}, \mathrm{O}_{3 C}$ and $\mathrm{O}_{\text {sub }}$. The calculated adsorption energy is shown in Table 2, and compared with the previous literature. The topology of each surface imposes the adsorption modes. The (110) two-fold coordinated sites is the most stable one with -0.30 $\mathrm{eV}$, and the corresponding subsurface position is slightly destabilized, with adsorption energy of $0.18 \mathrm{eV}$. The trend is similar to that found in ref. [13], and is preserved if no $\mathrm{U}$ parameter is considered. The (001) termination stabilizes subsurface positions by $-0.05 \mathrm{eV}$ and the (100) surface by $0.06 \mathrm{eV}$. 
Table 2 Calculated adsorption energies, $\mathrm{E}_{\mathrm{b}}(\mathrm{eV})$, for $\mathrm{H}$ adsorption on the rutile $\mathrm{TiO}_{2}$ (001), (100) and (110) surface.

\begin{tabular}{rlll}
\hline Types & $(001)$ & $(100)$ & $(110)$ \\
& DFT+U & DFT+U & DFT+U/Ref[13] \\
$1 \mathrm{H}: \mathrm{O}_{2 \mathrm{C}}$ & 0.43 & & $-0.30 /-0.23$ \\
$1 \mathrm{H}: \mathrm{O}_{3 \mathrm{C}}$ & -0.03 & 0.14 & $0.33 / 0.44$ \\
$1 \mathrm{H}: \mathrm{O}_{\text {sub }}$ & -0.05 & 0.06 & $0.18 / 0.40$ \\
\hline
\end{tabular}

\subsection{Diffusion of hydrogen on rutile $\mathrm{TiO}_{2}$ surfaces (001),}

\section{(100), (110)}

The pathways and structures for hydrogen diffusion from surface to subsurface are reported in Table 3 and Fig. 2, where the starting, transition state, and final structures are shown together with the energy profile. The path for hydrogen diffusion on rutile (001) is shown in Fig. 2 (a). In the starting structures, the $\mathrm{H}$ is attached to $\mathrm{O}_{3 \mathrm{C}}$ with an $\mathrm{O}-\mathrm{H}$ of $1.00 \AA$. In the transition state, one $\mathrm{H}$ migrates from $\mathrm{O}_{3 C}$ to $\mathrm{O}_{\text {sub }}$. In the final structure, one $\mathrm{H}$ is bound to Osub. The energy difference $(\Delta \mathrm{E})$ between the final and initial species of this migration process is $-0.02 \mathrm{eV}$. The activation barrier (Eact) is $0.39 \mathrm{eV}$, indicating possibly reversible migration. There is another pathway for $\mathrm{H}$

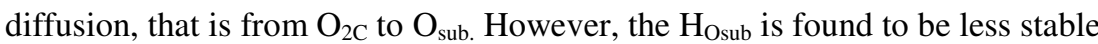
than the $\mathrm{H}_{\mathrm{O} 2 \mathrm{C}}$, by $0.48 \mathrm{eV}$. The distance between these two atoms is long, $2.816 \AA$, and the $\mathrm{OH}$ needs to rotate in this case, making this pathway unlikely So we consider the $\mathrm{H}$ diffusion from $\mathrm{O}_{3 \mathrm{C}}$ to $\mathrm{O}_{\text {sub }}$.

The adsorption energies with bigger unit cells were also calculated, and are shown in Table S1. The results indicate that with bigger unit cells (2x2) and lower coverage (0.25 ML), the hydrogen adsorption is stronger than higher coverage (1 ML), but the adsorption trend among (001), (100) and (110) is maintained. For the $\mathrm{H}$ diffusion on (100) (Fig. 2 (b)), one $\mathrm{H}$ is bound to $\mathrm{O}_{3 C}$ of the surface, then migrates to the subsurface region to form a bond with the $\mathrm{O}_{\text {sub }}$. In this process, the calculated $\Delta \mathrm{E}$ is $-0.08 \mathrm{eV}$, and the activation barrier of $\mathrm{H}$ migration is $0.79 \mathrm{eV}$, indicating that $\mathrm{H}$ diffusion to the subsurface region is an activated process, thermodynamically slightly favorable. For (110) it was shown previously that the direct 

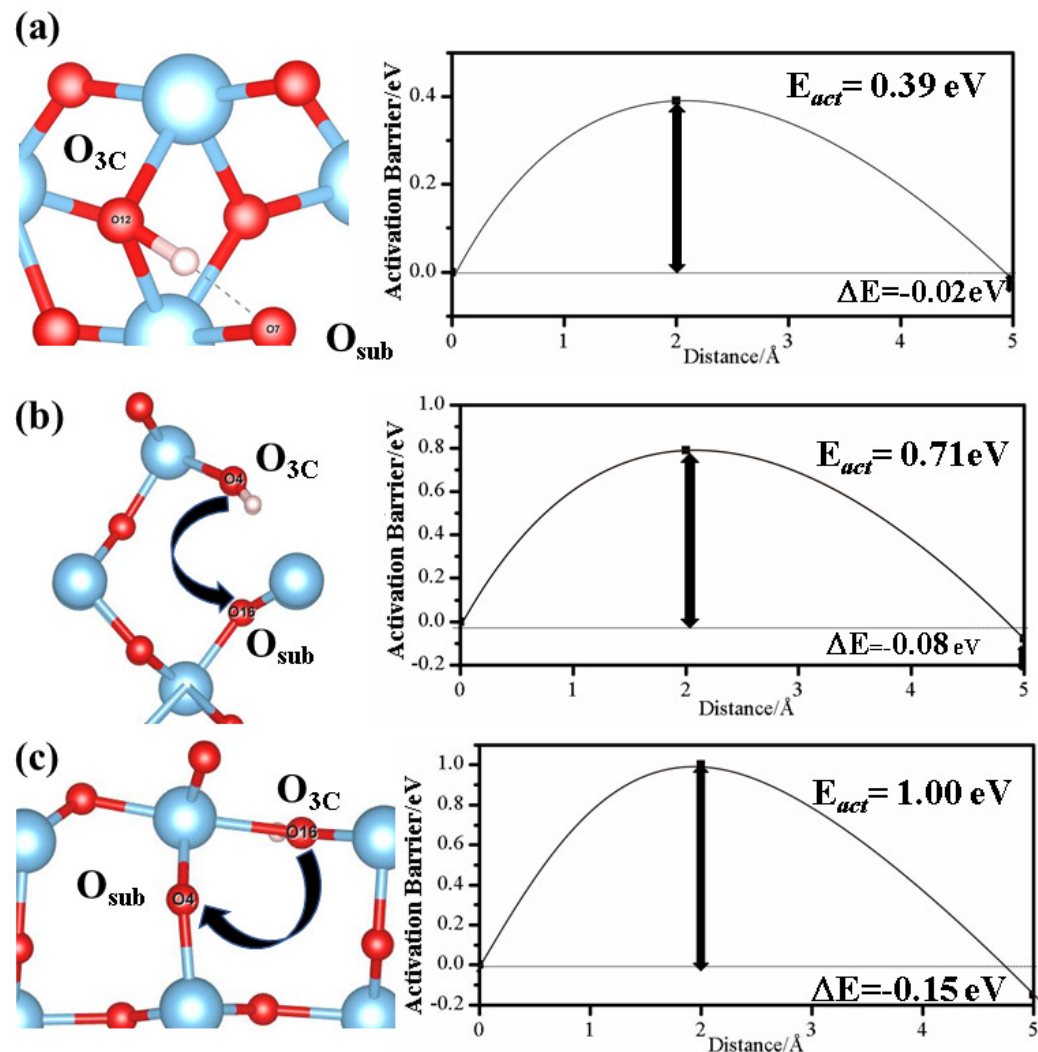

Fig. 2 Schematic structures of TS as well as energy profile in the diffusion process on three rutile $\mathrm{TiO}_{2}$ facets are shown. $\mathrm{E}_{\text {act }}$ indicates the activation barrier. $\Delta E$ is the reaction energy. (a), (b) and (c) are for (001), (100) and (110) respectively.

migration $\mathrm{O}_{2 C}$ to subsurface was more energetic [47]. Fig. 2(c) displays the diffusion of $\mathrm{H}$ from $\mathrm{O}_{3 C}$ to the subsurface site. In this process, the final structure is more stable than the initial one with an energy difference of $-0.15 \mathrm{eV}$. However, the higher barrier $(1.00 \mathrm{eV})$ makes this process less likely to occur compared to other terminations. The value of $1.00 \mathrm{eV}$ is consistent with the previous reports (1.11 eV [13], $0.93 \mathrm{eV}$ [47]). The small difference can come from the different coverage, layers and sizes, and the use of GGA+U in the present work.

We also calculated the energy profile without $U$ correction (values in brackets of Table 3). The trend is the same as that when $U$ correction was applied. The activation barriers are higher without $U$ correction, while the thermodynamic energies change very slightly. 
Table 3 Energy profile for the hydrogen diffusion process on three rutile facets. $\Delta \mathrm{E}$ is the reaction energy. Eact indicates the activation barrier. Values in brackets are without $\mathrm{U}$ correction.

\begin{tabular}{llll}
\hline & $(001)$ & $(100)$ & $(110)$ \\
Initial state & $0.00(0.00)$ & $0.00(0.00)$ & $0.00(0.00)$ \\
$\mathrm{E}_{a c t}$ & $0.39(0.43)$ & $0.71(0.79)$ & $1.00(1.03)$ \\
$\Delta \mathrm{E}$ & $-0.02(-0.03)$ & $-0.08(-0.09)$ & $-0.15(-0.19)$ \\
\hline
\end{tabular}

\section{3 $\mathrm{H}$ diffusion in the bulk $\mathrm{TiO}_{2}$}

Once in the subsurface, $\mathrm{H}$ binds to a lattice $\mathrm{O}$ forming a hydroxyl group, that can migrate inside the structure. We have considered three diffusion paths of the $\mathrm{H}$ atom in the bulk (See Fig. 3). These diffusion paths are described in detail below and the activation barriers are indicated in Table 4. The $\mathrm{H}$ in the bulk is thus bound to $\mathrm{O}_{3 C}$, with a distance $0.998 \AA$ for the O-H bond. Three migration paths, involving other $\mathrm{O}_{3 C}$ sites, are considered, with an energy difference $(\Delta \mathrm{E})$ of zero. The $\mathrm{H}$ diffuses through a small cavity with activation barrier ranging from $0.32 \mathrm{eV}$ to $0.97 \mathrm{eV}$. The first path involves the migration from one cavity to a neighboring cavity, see Fig. 3(b), and the activation energy is $0.97 \mathrm{eV}$. The most likely migration pathway is the migration of $\mathrm{H}$ from $\mathrm{O}_{b}$ to $\mathrm{O}_{d}$ (see Fig. 3(c)), with the lowest energy barrier, only $0.32 \mathrm{eV}$. The two $\mathrm{O}$ atoms involved in this process are positioned directly opposite to each other within the same cavity, forming an H-bond, which favors $\mathrm{H}$ migration. For the $\mathrm{H}$ diffusion from $\mathrm{O}_{d}$ to $\mathrm{O}_{c}$, the barrier is $0.53 \mathrm{eV}$, Fig. 3 (d). It involves in the same cavity between two $\mathrm{O}$ sites without $\mathrm{H}-$ bond.

Table 4 Activation Barriers $\mathrm{E}_{a c t}(\mathrm{eV})$, for Atomic Hydrogen diffusion in the $\mathrm{TiO}_{2}$ bulk, see Fig. 3.

\begin{tabular}{ll}
\hline Diffusion type & $\mathrm{E}_{a c t}$ \\
Path 1: $\mathrm{O}_{\mathrm{a}} \rightarrow \mathrm{O}_{\mathrm{b}}$ & 0.97 \\
Path 2: $\mathrm{O}_{\mathrm{b}} \rightarrow \mathrm{O}_{\mathrm{d}}$ & 0.32 \\
Path 3: $\mathrm{O}_{\mathrm{d}} \rightarrow \mathrm{O}_{\mathrm{c}}$ & 0.53 \\
\hline
\end{tabular}


(a)

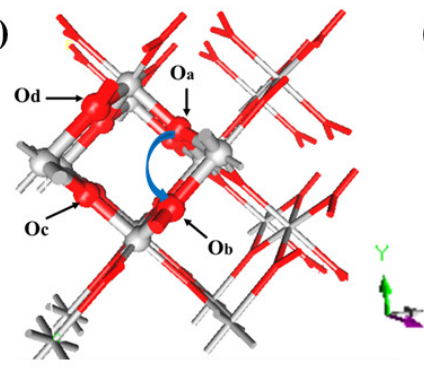

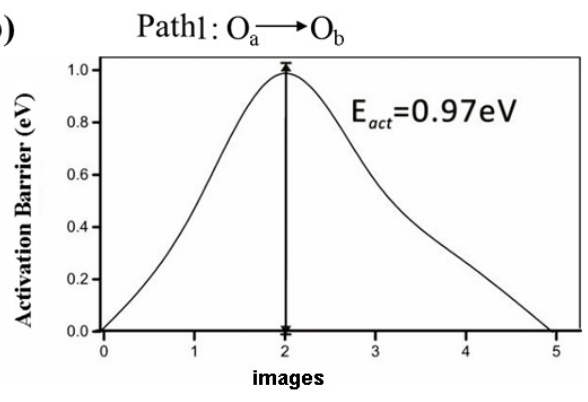

(d) Path 3: $\mathrm{O}_{\mathrm{d}} \longrightarrow \mathrm{O}_{\mathrm{c}}$
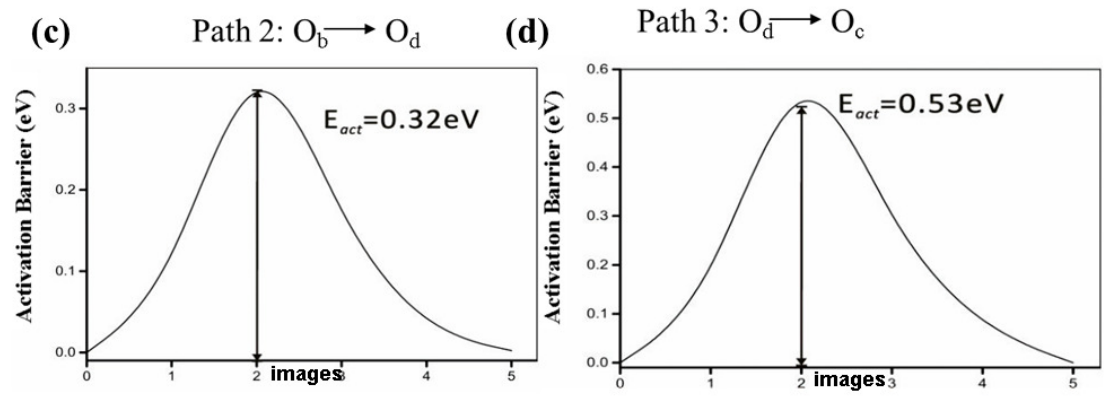

Fig. 3 (a) A cavity of the rutile $\mathrm{TiO}_{2}$ bulk (a $2 \times 2 \times 2$ supercell was used). (b) Path 1: $\mathrm{H}$ migrates in the bulk from $\mathrm{O}_{a}$ to $\mathrm{O}_{b}$; (c) Path 2: $\mathrm{H}$ migrates in the bulk from $\mathrm{O}_{b}$ to $\mathrm{O}_{d}$; (d)Path 3: $\mathrm{H}$ migrates in the bulk from $\mathrm{O}_{d}$ to $\mathrm{O}_{c}$. Grey, red represent the titanium and oxygen atoms respectively.

Some of us have studied the $\mathrm{H}$ insertion into rutile in deeper subsurface layers (110) [13], with the energy barriers of 1.02-1.13 eV, which is consistent with the present value of $0.97 \mathrm{eV}$ for the migration between neighboring cavities.

\subsection{Electronic Structure}

When an $\mathrm{H}$ adsorbs on an oxygen site, there is always a charge transfer of one electron with formation of a proton $\mathrm{H}^{+}$bound to an $\mathrm{O}$, and one electron that is transferred to the empty $\mathrm{Ti} 3 \mathrm{~d}$ states, as found experimentally [48] and theoretically [41].

This can be visualized by the density of states (DOS). Thus we studied the partial DOS Ti states for the hydrogen adsorption on the oxygen site of the surface $\left(\mathrm{O}_{\text {sur }}\right)$ and also subsurface $\mathrm{O}_{\text {sub }}$ (See Fig. 4, Fig. S1-S3). Compared with the bare slab, it is clearly indicated that there is a new $\mathrm{Ti}^{3+}$ peak near the Fermi energy for hydrogenated slabs. This electronic feature is found for the three model slabs 
used, and also for the bulk system (Fig. S3).
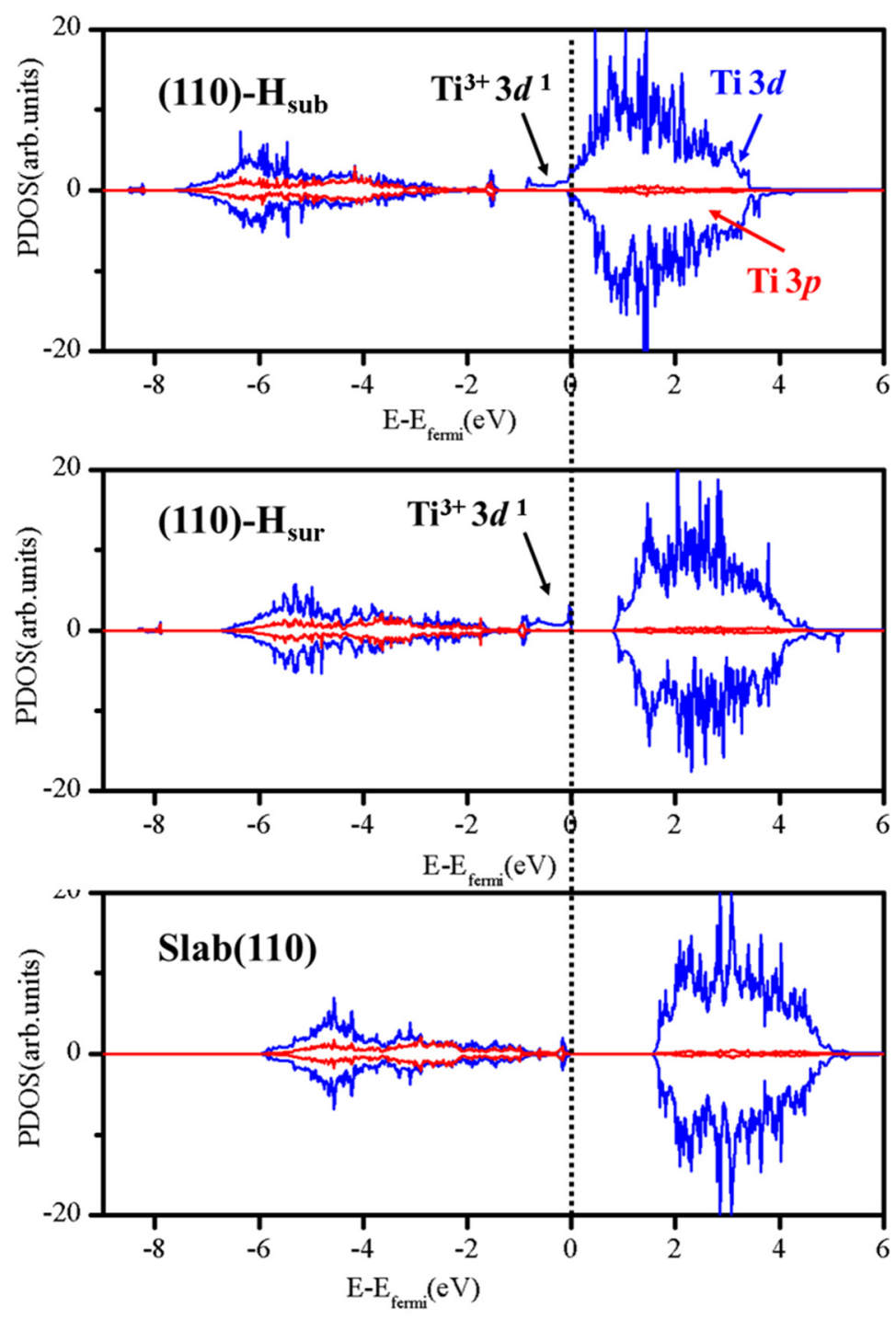

Fig. 4 PDOS of Ti 3d and 3p states obtained at the GGA+U level for slab (110) and hydrogenated species $\left(\mathrm{H}_{\text {sur }}\right.$ and $\left.\mathrm{H}_{s u b}\right)$ in the diffusion process. The localized $\mathrm{Ti}^{3+} 3 \mathrm{~d}^{1}$ state is marked by an arrow. PDOS of Ti $3 d$ and $3 p$ states for (100), (001) and bulk are provided in SI (Fig. S1-S3)

An analysis of the spin density of the hydrogenated species has been performed (see Fig. 5, Fig. S4 and S5), which shows the location of unpaired electrons. The Bader spin analysis is reported (Table 5, Table S3, S4). One Ti was reduced for every facet. For (110), for initial state and TS, the five-fold Ti 
on the surface was reduced, with around 0.97-0.99 $|e|$. However, when H binds to the subsurface $\mathrm{O}$, the subsurface Ti was mainly reduced, with 0.60 lel (Table 5 and Fig. 5), which is also found in the diffusion process of (001) (Table S1 and Fig. S4). For (100), the subsurface Ti was always reduced dur- ing the process with the integrated spin density varying from 0.84 to $0.98 \mathrm{lel}$ (Table S2 and Fig.S5). One Ti was also reduced when $\mathrm{H}$ bound with $\mathrm{O}$ in the bulk, with 0.92 |el (Table S2).

Table 5 Bader charges in lel of selected Ti, $\mathrm{O}$ and $\mathrm{H}$ atoms for the hydrogen diffusion from surface to subsurface process on rutile $\mathrm{TiO}_{2}(110)$. Inside the brackets indicates the integrated Bader spin.

\begin{tabular}{lllll}
\hline & & \multicolumn{3}{c}{$(110)$} \\
atoms & slab & Hsur & TS & Hsub \\
TisC-sur & $2.02(0.00)$ & $1.79(0.99)$ & $1.81(0.97)$ & $1.98(0.25)$ \\
Tisub & $2.04(0.00)$ & $2.04(0.01)$ & $2.06(0.02)$ & $1.92(0.60)$ \\
$\mathrm{O}$ & $-1.05(0.00)$ & $-1.10(0.00)$ & $-1.11(0.00)$ & $-1.22(0.00)$ \\
$\mathrm{O}$ & $-1.05(0.00)$ & $-1.26(0.00)$ & $-1.25(0.00)$ & $-1.08(0.00)$ \\
$\mathrm{H}$ & & $0.65(0.00)$ & $0.62(0.00)$ & $0.62(0.00)$ \\
\hline
\end{tabular}

There is a debate regarding the location of reduced $\mathrm{Ti}^{3+}$. The previous studies like [41] and also [49], show that it can be influenced by the computational settings such as functional, and Hubbard correction U. Moreover, the chemical environment of the $\mathrm{OH}$ group might also affect the position of the reduced $\mathrm{Ti}$, e.g. in reference [49], the hydrogen is bound to bridging oxygen $\left(\mathrm{O}_{2 C}\right)$, while in our process, the hydrogen is bound to threecoordinated oxygen $\left(\mathrm{O}_{3 C}\right)$. In the present work we have chosen to focus on the energetics of the diffusion process rather than on the electron localization problem.

In addition, although in the paper [49], the most stable site for the location of excess electron occurs in the second layer directly beneath the $\mathrm{Ti}_{5 C}$ row, there is still possibilities for the location at the surface $\mathrm{Ti}(0.1 \mathrm{eV}$ less stable) and third layer $(0.2 \mathrm{eV}$ less stable). The energy differences $(0.1 \mathrm{eV}-0.2 \mathrm{eV})$ are not big. We have unsuccessfully tried to locate the electron in the subsurface position, and it always converges to the surface position. Assuming that the energetic effect is constant, the trends of the diffusion barriers should not be affected by the site where the excess electrons are 


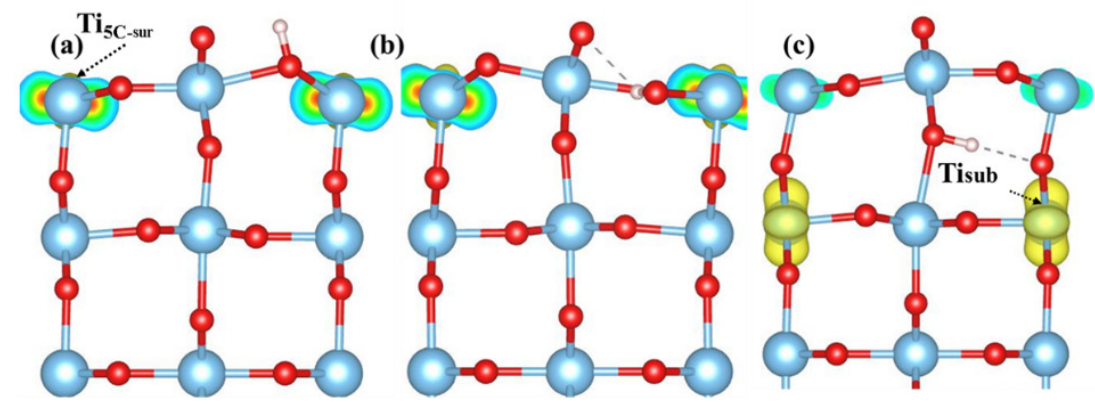

Fig. 5 Spin density of initial and final species during the diffusion process for (110)

surface, which indicates the distribution of unpaired electrons (isosurface level=0.01).

Data for (001) and (100) and bulk are provided in SI (Fig. S4 and S5).

\subsection{Analysis of reactivity}

Based on our results in Table 6 and Fig. 2, it is clearly seen that the diffusion of $\mathrm{H}$ on three surfaces behaves significantly different. We found that the activation barrier is linearly related to the distance between the $\mathrm{O}$ of the surface and subsurface $\mathrm{O}_{\text {sub }}$ in the bare slabs (Fig. 6). Moreover, when we analyze the structures of the transition states (TS in Fig. 2), it was found that for (100) and (110), the O-H needs to rotate in order to be bound to $\mathrm{O}_{s u b}$. For (001), the $\mathrm{H}$ can diffuse from $\mathrm{O}_{3 C}$ to $\mathrm{O}_{\text {sub }}$ directly. Thus in these former two processes, it needs higher activation barrier than (001). The same trend is observed in the absence of $U$ parameter, although with a worse linear correlation.

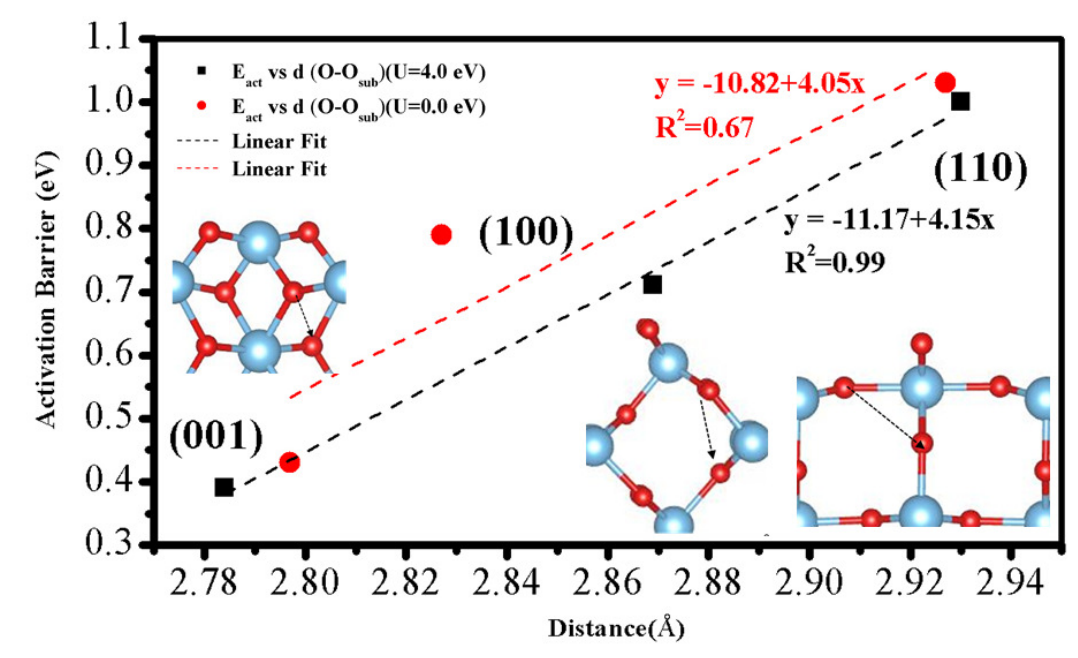

Fig. 6 The activation barrier $\left(\mathrm{E}_{a c t}\right)$ as a function of the distance between the $\mathrm{O}$ of the 
Table 6 The activation barrier $\mathrm{E}_{\text {act }}(\mathrm{eV})$ as a function of the distance difference $\mathrm{d}(\mathrm{O}-$ Osub) (A) between the $\mathrm{O}$ of the surface and subsurface of the bare slab with and without $\mathrm{U}$ correction.

\begin{tabular}{lll}
\hline & $\mathrm{d}-\left(\mathrm{O}-\mathrm{O}_{\text {sub }}\right)(\AA)$ & Activation barrier $\left(\mathrm{E}_{a c t}\right)(\mathrm{eV})$ \\
Slabs & & \\
$(001)-(\mathrm{U}=4.0 \mathrm{eV})$ & 2.784 & 0.39 \\
$(001)-(\mathrm{U}=0.0 \mathrm{eV})$ & 2.797 & 0.43 \\
$(100)-(\mathrm{U}=4.0 \mathrm{eV})$ & 2.869 & 0.71 \\
$(100)-(\mathrm{U}=0.0 \mathrm{eV})$ & 2.827 & 0.79 \\
$(101)-(\mathrm{U}=4.0 \mathrm{eV})$ & 2.930 & 1.00 \\
$(101)-(\mathrm{U}=0.0 \mathrm{eV})$ & 2.927 & 1.03 \\
\hline
\end{tabular}

\subsection{Isotopic substitution and thermal corrections}

In order to estimate the probability of tunneling effects we have considered the isotopic substitution of $\mathrm{H}$ by Deuterium for the (110) termination. The initial, transition and final structures were reoptimized. We investigated two pathways (Fig. 7, (a-b) and (c-d)) to make a comparison. For process (a-b) there is only one $\mathrm{H}$ in the cell that diffuses from $\mathrm{O}_{3 C}$ to $\mathrm{O}_{s u b}$. For process (c- d), one additional $\mathrm{H}$ is present on the $\mathrm{O}_{2 C}$ site, and $\mathrm{H}$ migrates from $\mathrm{O}_{3 C}$ to $\mathrm{O}_{s u b}$. The results show that the energy differences between initial and final structures are slightly affected by the deuteration, (Fig. 7, (b) and (e)), decreasing by $-0.09 \mathrm{eV}$, whereas the energetic barriers are increased by 0.08 $\mathrm{eV}$, as expected from the higher weight of $\mathrm{D}$ compared to $\mathrm{H}$. The two paths are also very similar as regards reaction and activation energies, despite the fact the the latter possesses a higher degree of reduction due to the presence of a second $\mathrm{H}$ atom. No significant isotopic substitution effect was found.

The ZPE correction was also calculated. The results (in Fig. 7, red lines) showed that there is no effect on reaction energy $(\Delta \mathrm{E})$, but the kinetic barrier is slightly reduced, by -0.04 to $-0.02 \mathrm{eV}$. The same behavior is observed for the other two surfaces, the details for (100) and (101) are provided in SI (Fig. S6S8)

\subsection{Substrate reduction effect}

The degree of reduction of the titania substrate has been studied by two sets of models schematized in Fig. 8. By adding or removing neutral species, such as water adsorption (no excess electron), $\mathrm{H}$ addition (each $\mathrm{H}$ adds one 
(a)

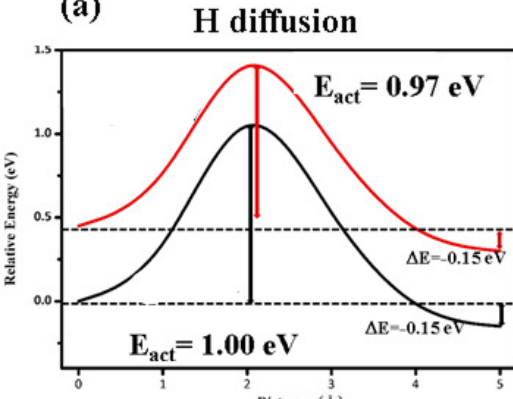

(c)

H diffusion

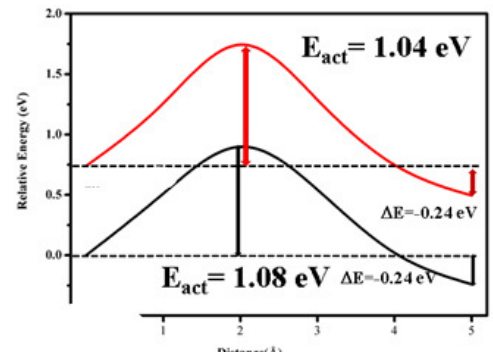

(b)

Fig. 7 Comparison of calculated reaction energies $(\Delta \mathrm{E})$ and activation barriers Eact for hydrogen and deuterium adsorption on the rutile (110) surfaces. In pathway (a) and (b), one $\mathrm{H}$ is present whereas in pathway (c) and (d) a second $\mathrm{H}$ is located on $\mathrm{O}_{2 \mathrm{C}}$. Zero Point Energy corrected profile (red line) and uncorrected (black line) are represented.

In this case we may expect additional interactions (local relaxations, $\mathrm{H}$ bonds...). Second, by modifying manually the number of electrons of the supercell, resulting in charged systems (VASP compensates the charge by a uniform background compensating charge). The transition states are just single points to keep the geometry and vacuum fixed, as they influence the energy of charged periodic systems, and the results have to be interpreted as rough trends rather than quantitative data. Thus, the resulting systems studied are:

a) neutral species

a1) the non-reduced surface (denoted as $0 \mathrm{e}$ ) by adsorbing $1 \mathrm{OH}^{-}$and 1

$\mathrm{H}^{+}$, corresponds to formal water adsorption with no electron transfer

a2) the reduced surface (denoted $1 \mathrm{e}$ ) by adsorbing $1 \mathrm{H}$ 
a3) the reduced surface (denoted $2 \mathrm{e}$ ) by adsorbing $2 \mathrm{H}$

a4) the reduced surface (denoted $3 \mathrm{e}$ ) by adsorbing $1 \mathrm{H}$ and removing 1 O.

b) charged systems: we add 0 e, 1 e, 2 e, 3 e electrons to the slab model.

The activation barriers of diffusion in case a are $1.18 \mathrm{eV}$ (neutral), 1.01 $\mathrm{eV}(1 \mathrm{e}), 1.08 \mathrm{eV}(2 \mathrm{e}), 0.76 \mathrm{eV}(3 \mathrm{e})$ respectively. The activation barriers of diffusion in case $\mathrm{b}$ are $1.37 \mathrm{eV}$ (neutral), $1.01 \mathrm{eV}(1 \mathrm{e}), 0.81 \mathrm{eV}(2 \mathrm{e}), 0.75 \mathrm{eV}$ (3 e) respectively. In both cases, the activation barrier of the diffusion on the surface with $0 \mathrm{e}$ is the highest, while with $3 \mathrm{e}$ is the lowest. There seems to be a trend relating high reduction state and low diffusion barrier. In realistic conditions where titania is partially reduced (presence of $\mathrm{H}$ impurities, oxygen vacancies), it is thus expected that migration of hydrogen to bulk positions is favored compared to stoichiometric compositions.

In order to gain more understanding in the reason behind such behavior, we perform a spin density analysis (Fig. S9) for the 8 transition states calculated. For the non-reduced surface (denoted as $0 \mathrm{e}$ ), there is no unpaired electrons. For other kinds of reduced surface (denoted as 1e, 2e, 3e), the number of Ti sites bearing electrons increase with the reduction degree. Thus, one excess electron results mainly in the population of the $\mathrm{Ti}_{5 C}$ site with a small contribution from a subsurface Ti, irrespective of the way it has been added. For more than one excess electrons, case a) results in a wider distribution of the spin over more Ti sites than case b). In all the situations both surface and subsurface Ti sites are involved. The reduction of subsurface Ti sites was already invoked by Jupille et al [51]. 


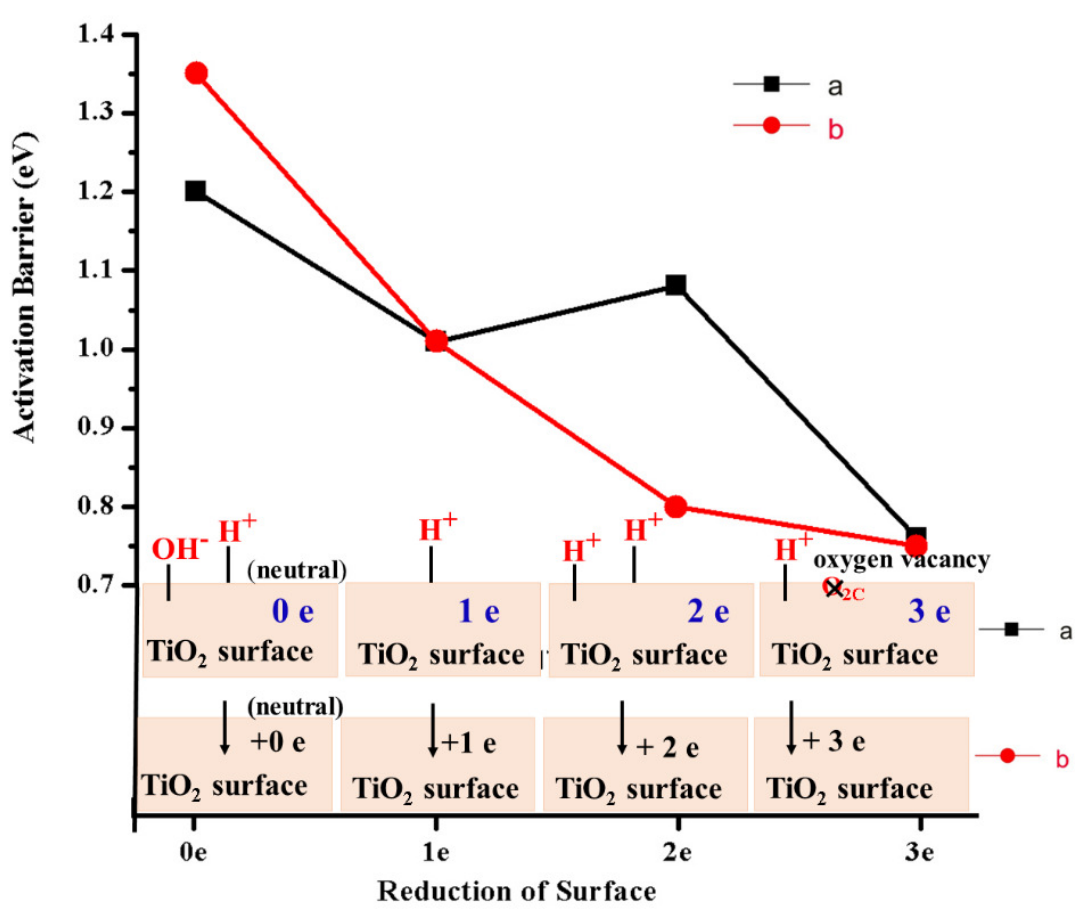

Fig. 8 The activation barriers on four kinds of reduced surfaces for hydrogen diffusion from surface to subsurface. The surfaces were reduced by two ways. (a): Adsorption of $\mathrm{H}$ or removing oxygen: Adsorption of $1 \mathrm{H}^{+}$and $1 \mathrm{OH}^{-}$(denoted as $0 \mathrm{e}$ ); Adsorption of $1 \mathrm{H}$ (denoted as $1 \mathrm{e}$ ); Adsorption of $2 \mathrm{H}$ (denoted as $2 \mathrm{e}$ ); Adsorption of $1 \mathrm{H}$ and removing $1 \mathrm{O}$ (denoted as $3 \mathrm{e}$ ); (b) Adding electrons to the system directly (by setting INCAR in VASP). The non-reduced surface (denoted as 0e); The reduced surface (denoted $1 \mathrm{e}$ ); The reduced surface (denoted 2e); The reduced surface (denoted 3e).

Based on Bader charge analysis results (Table S4), we find that Ti and $\mathrm{H}$ lose electrons, and $\mathrm{O}$ gain electrons. Interestingly, when the surfaces are more reduced, the numbers of electrons lost by $\mathrm{Ti}_{a}$ and $\mathrm{Ti}_{b}$ are fewer. That means more electrons are located in $\mathrm{Ti}_{a}$ and $\mathrm{Ti}_{b}$ when surfaces are more reduced. So $\mathrm{Ti}_{a}$ and $\mathrm{Ti}_{b}$ can attract $\mathrm{H}$, the transition states are stabilized. In addition, we also find correlation between the energy barriers and the distances between $\mathrm{Ti}_{b}$ and $\mathrm{H}$ (See Fig. S9 (c)), and also the correction between $\mathrm{d}$ and reduction of the surface (See Fig. S9 (d)). The presence of excess electrons in Ti sites seems to i) decrease the electrostatic repulsion between $\mathrm{Ti}$ and $\mathrm{H}$ and ii) increase the distance between $\mathrm{Ti}_{5 C}$ and $\mathrm{H}$ in the TS, both resulting in a decrease of the TS 


\section{Conclusion}

The mechanisms for diffusion from surface into the subsurface over three rutile $\mathrm{TiO}_{2}$ surfaces and bulk are investigated with $\mathrm{PBE}+\mathrm{U}$ calculations. The topology structures have strong effects on kinetic barriers, while slightly change the themodynamic energies. The activation barriers of diffusion are $0.39 \mathrm{eV}, 0.79 \mathrm{eV}, 1.00 \mathrm{eV}$ for surface (001) (100) and (110) respectively. Our results indicate that (001) shows the most favorable path for (reversible) diffusion. Once in the bulk, $\mathrm{H}$ could diffuse easily inside cavities with barriers as low as $0.32 \mathrm{eV} \mathrm{eV}$, whereas migration to other cavities is more costly, 0.96 $\mathrm{eV}$. We observe a linear correlation between the activation energy and the distance $\mathrm{O}_{\text {surf }}-\mathrm{O}_{\text {sub }}$ in the bare slab.

For rutile (110), isotopic substitution by deuterium results in slight changes in the kinetic barriers of $+0.04 \mathrm{eV}$ compared to hydrogen. The presence of other surface $\mathrm{H}$ atom in $\mathrm{O}_{2 C}$ position resulted in a slight increase of the activation barrier $(1.08 \mathrm{eV}$ vs $1.00 \mathrm{eV})$ and more negative reaction energy $(-0.24 \mathrm{eV}$ vs $-0.15 \mathrm{eV})$. Thermal effects also affected slightly the energetic profiles.

We found that the substrate reduction degree has a moderate impact in the activation barriers, decreasing from more than $1 \mathrm{eV}$ down to $0.8 \mathrm{eV}$. The reason could be the increase of the Ti-H distance in the TS that results in a stabilization and thus a decrease in the activation energy.

In summary, our results clearly indicate the complexity of the surface to subsurface hydrogen diffusion process, and suggest that controlling the topology and the reduction degree are valid strategies to tune such process.

\section{Declaration of Competing Interest}

The authors declare that they have no known competing financial interests or personal relationships that could have appeared to influence the work reported in this paper.

\section{CRediT authorship contribution statement}




\section{Acknowledgements}

Authors acknowledge Scienomics for the courtesy license of the MAPS program used in the construction of the slab models, and COST Action CA18234. Dr. B. Diawara is acknowledged for the Modelview program. This work was performed using HPC resources from GENCI- CINES/IDRIS (Grant 2020- x2020082131 and 2021- x2021082131).

\section{References}

[1] Crossland, E.J., Noel, N., Sivaram, V., Leijtens, T., Alexander-Webber, J.A., Snaith, H.J.: Mesoporous $\mathrm{TiO}_{2}$ single crystals delivering enhanced mobility and optoelectronic device performance. Nature 495(7440), 215219 (2013)

[2] Vila,ca, G., Jousseaume, B., Mahieux, C., Belin, C., Cachet, H., Bernard, M.C., Vivier, V., Toupance, T.: Tin dioxide materials chemically modified with trialkynylorganotins: functional nanohybrids for photovoltaic applications. Advanced Materials 18(8), 1073-1077 (2006)

[3] Zhou, X., Wang, Z., Xia, X., Shao, G., Homewood, K., Gao, Y.: Synergistic cooperation of rutile $\mathrm{TiO}_{2}\{002\},\{101\}$, and $\{110\}$ facets for hydrogen sensing. ACS applied materials \& interfaces 10(33), 28199-28209 (2018)

[4] Varghese, O.K., Gong, D., Paulose, M., Ong, K.G., Dickey, E.C., Grimes, C.A.: Extreme changes in the electrical resistance of titania nanotubes with hydrogen exposure. Advanced Materials 15(7-8), 624-627 (2003)

[5] Tada, H., Fujishima, M., Kobayashi, H.: Photodeposition of metal sulfide quantum dots on titanium (iv) dioxide and the applications to solar energy conversion. Chemical Society Reviews 40(7), 4232-4243 (2011)

[6] Maeda, Y., Iizuka, Y., Kohyama, M.: Generation of oxygen vacancies at $\mathrm{a}$ au/ $/ \mathrm{TiO}_{2}$ perimeter interface during co oxidation detected by in situ electrical conductance measurement. Journal of the American Chemical Society 135(2), 906-909 (2013) 
[7] Sun, C., Jia, Y., Yang, X.-H., Yang, H.-G., Yao, X., Lu, G.Q., Selloni, A., Smith, S.C.: Hydrogen incorporation and storage in well-defined nanocrystals of anatase titanium dioxide. The Journal of Physical Chemistry C 115(51), 25590-25594 (2011)

[8] Joo, J.B., Dillon, R., Lee, I., Yin, Y., Bardeen, C.J., Zaera, F.: Promotion of atomic hydrogen recombination as an alternative to electron trapping for the role of metals in the photocatalytic production of $\mathrm{H}_{2}$. Proceedings of the National Academy of Sciences 111(22), 7942-7947 (2014)

[9] Liu, X., Zhu, G., Wang, X., Yuan, X., Lin, T., Huang, F.: Progress in black titania: a new material for advanced photocatalysis. Advanced Energy Materials 6(17), 1600452 (2016)

[10] Mao, W., Wilde, M., Ogura, S., Chen, J., Fukutani, K., Matsuzaki, H., Terai, T.: Hydrogen-accelerated phase transition and diffusion in $\mathrm{TiO}_{2}$ thin films. The Journal of Physical Chemistry C 122(40), 23026-23033(2018)

[11] Li, X., Lin, J., Li, L., Huang, Y., Pan, X., Collins, S.E., Ren, Y., Su, Y., Kang, L., Liu, X., et al.: Controlling $\mathrm{CO}_{2}$ hydrogenation selectivity by metal-supported electron transfer. Angewandte Chemie International Edition 59(45), 19983-19989 (2020)

[12] Diebold, U.: The surface science of titanium dioxide. Surface science reports 48(5-8), 53-229 (2003)

[13] Yin, X.-L., Calatayud, M., Qiu, H., Wang, Y., Birkner, A., Minot, C., W“oll, C.: Diffusion versus desorption: complex behavior of $\mathrm{h}$ atoms on an oxide surface. Chem Phys Chem 9(2), 253-256 (2008)

[14] Du, Y., Petrik, N.G., Deskins, N.A., Wang, Z., Henderson, M.A., Kimmel, G.A., Lyubinetsky, I.: Hydrogen reactivity on highly hydroxylated $\mathrm{TiO}_{2}$ (110) surfaces prepared via carboxylic acid adsorption and photolysis. Physical Chemistry Chemical Physics 14(9), 3066-3074 (2012)

[15] Kreuer, K.D.: Proton-conducting oxides. Annual Review of Materials Research 33(1), 333-359 (2003)

[16] Chen, X., Liu, L., Peter, Y.Y., Mao, S.S.: Increasing solar absorption for photocatalysis with black hydrogenated titanium dioxide nanocrystals. Science 331(6018), 746-750 (2011)

[17] Hu, Y.H.: A highly efficient photocatalyst—hydrogenated black $\mathrm{TiO}_{2}$ for 
the photocatalytic splitting of water. Angewandte Chemie International Edition 51(50), 12410-12412 (2012)

[18] Leshuk, Tim, Parviz, Roozbeh, Robert A and Gu, Frank, Photocatalytic activity of hydrogenated $\mathrm{TiO}_{2}$. ACS applied materials 5, (6), 18921895(2013)

[19] Wang, X., Zhang, S., Xie, Y., Wang, H., Yu, H., Shen, Y., Li, Z., Zhang, S., Peng, F.: Branched hydrogenated $\mathrm{TiO}_{2}$ nanorod arrays for improving photocatalytic hydrogen evolution performance under simulated solar light. International Journal of Hydrogen Energy 41(44), 20192-20197 (2016)

[20] Xia, T., Chen, X.: Revealing the structural properties of hydrogenated black Ti nanocrystals. Journal of Materials Chemistry A 1(9), 2983-2989 (2013)

[21] Yan, Y., Han, M., Konkin, A., Koppe, T., Wang, D., Andreu, T., Chen, G., Vetter, U., Morante, J.R., Schaaf, P.: Slightly hydrogenated $\mathrm{TiO}_{2}$ with enhanced photocatalytic performance. Journal of Materials Chemistry A 2(32), 12708-12716 (2014)

[22] Zhang, D., Yang, M., Dong, S.: Hydroxylation of the rutile $\mathrm{TiO}_{2}(110)$ surface enhancing its reducing power for photocatalysis. The Journal of Physical Chemistry C 119(3), 1451-1456 (2015)

[23] Nadeem, I.M., Treacy, J.P., Selcuk, S., Torrelles, X., Hussain, H., Wilson, A., Grinter, D.C., Cabailh, G., Bikondoa, O., Nicklin, C., et al.: Water dissociates at the aqueous interface with reduced anatase $\mathrm{TiO}_{2}(101)$. The journal of physical chemistry letters 9(11), 3131-3136 (2018)

[24] Liu, L., Wang, Z., Pan, C., Xiao, W., Cho, K.: Effect of hydrogen

on $\mathrm{O}_{2}$ adsorption and dissociation on a $\mathrm{TiO}_{2}$ anatase (001) surface.

ChemPhysChem 14(5), 996-1002 (2013)

[25] Aschauer, U., Selloni, A.: Hydrogen interaction with the anatase $\mathrm{TiO}_{2}$ (101) surface. Physical Chemistry Chemical Physics 14(48), 16595-16602 (2012)

[26] Islam, M.M., Calatayud, M., Pacchioni, G.: Hydrogen adsorption and diffusion on the anatase $\mathrm{TiO}_{2}$ (101) surface: a first-principles investigation. The Journal of Physical Chemistry C 115(14), 6809-6814 (2011) 
[27] Calatayud, M., Minot, C.: Effect of relaxation on structure and reactivity of anatase (llll $\left.\begin{array}{lll}0 & 0\end{array}\right)$ and $\left(\begin{array}{lll}0 & 0 & 1\end{array}\right)$ surfaces. Surface science 552(1-3), 169-179 (2004)

[28] Lei, M., Coh, S.: Hydrogen plasma favored modification of anatase $\mathrm{TiO}_{2}$ (001) surface with desirable water splitting performance. Physical Review Materials 4(7), 075801 (2020)

[29] Enevoldsen, G.H., Pinto, H.P., Foster, A.S., Jensen, M.C., Hofer, W.A., Hammer, B., Lauritsen, J.V., Besenbacher, F.: Imaging of the hydrogen subsurface site in rutile $\mathrm{TiO}_{2}$. Physical review letters 102(13), 136103(2009)

[30] Lu, Y., Yin, W.-J., Peng, K.-L., Wang, K., Hu, Q., Selloni, A., Chen, F.R., Liu, L.-M., Sui, M.-L.: Self-hydrogenated shell promoting photocatalytic $\mathrm{H}_{2}$ evolution on anatase $\mathrm{TiO}_{2}$. Nature communications 9(1), 1-9 (2018)

[31] Ohashi, Y., Nagatsuka, N., Ogura, S., Fukutani, K.: Hydrogen distribution and electronic structure of $\mathrm{TiO}_{2}$ (110) hydrogenated with lowenergy hydrogen ions. The Journal of Physical Chemistry C 123(16), 10319-10324 (2019)

[32] Nagatsuka, N., Wilde, M., Fukutani, K.: Hydrogenation and hydrogen diffusion at the anatase $\mathrm{TiO}_{2}$ (101) surface. The Journal of chemical physics 152(7), 074708 (2020)

[33] Morris Hotsenpiller, P., Bolt, J., Farneth, W., Lowekamp, J., Rohrer, G.: Orientation dependence of photochemical reactions on $\mathrm{TiO}_{2}$ surfaces. The Journal of Physical Chemistry B 102(17), 3216-3226 (1998)

[34] Kislov, N., Lahiri, J., Verma, H., Goswami, D.Y., Stefanakos, E., Batzill, M.: Photocatalytic degradation of methyl orange over single crystalline zno: orientation dependence of photoactivity and photostability of Zno. Langmuir 25(5), 3310-3315 (2009)

[35] Kyriakou, G., Davidson, E.R., Peng, G., Roling, L.T., Singh, S., Boucher, M.B., Marcinkowski, M.D., Mavrikakis, M., Michaelides, A., Sykes, E.C.H.: Significant quantum effects in hydrogen activation. ACS nano 8(5), 4827-4835 (2014)

[36] Walle, L., Borg, A., Uvdal, P., Sandell, A.: Probing the influence from 
residual ti interstitials on water adsorption on $\mathrm{TiO}_{2}$ (110). Physical Review B 86(20), 205415 (2012)

[37] Perdew, J.P., Burke, K., Ernzerhof, M.: Generalized gradient approximation made simple. Physical review letters 77(18), 3865 (1996)

[38] Bl"ochl, P.E.: Projector augmented-wave method. Physical review B 50(24), 17953 (1994)

[39] Kresse, G., Hafner, J.: Ab initio molecular dynamics for liquid metals. Physical Review B 47(1), 558 (1993)

[40] Dudarev, S., Botton, G., Savrasov, S., Humphreys, C., Sutton, A.:

Electron energy loss spectra and the structural stability of nickel oxide: An lsda+ u study. Physical Review B 57(3), 1505 (1998)

[41] Finazzi, E., Di Valentin, C., Pacchioni, G., Selloni, A.: Excess electron states in reduced bulk anatase $\mathrm{TiO}_{2}$ : comparison of standard gga, gga+ u, and hybrid dft calculations. The Journal of chemical physics 129(15), 54113 (2008)

[42] van de Walle, A., Ceder, G.: Automating first-principles phase diagram calculations. Journal of Phase Equilibria 23(4), 348-359 (2002)

[43] Suzuki, S., Fukui, K.-i., Onishi, H., Iwasawa, Y.: Hydrogen adatoms on $\mathrm{TiO}_{2}(110)-(1 \diamond 1)$ characterized by scanning tunneling microscopy and electron stimulated desorption. Physical Review Letters 84(10), 2156(2000)

[44] Kunat, M., Burghaus, U., W*oll, C.: The adsorption of hydrogen on the rutile $\mathrm{TiO}_{2}$ (110) surface. Physical Chemistry Chemical Physics 6(16), 4203-4207 (2004)

[45] Henkelman, G., J'onsson, H.: Improved tangent estimate in the nudged elastic band method for finding minimum energy paths and saddle points. The Journal of chemical physics 113(22), 9978-9985 (2000)

[46] Henkelman, G., J'onsson, H.: A dimer method for finding saddle points on high dimensional potential surfaces using only first derivatives. The Journal of chemical physics 111(15), 7010-7022 (1999)

[47] Calatayud, M., Yin, X.-L., Qiu, H., Wang, Y., Birkner, A., Minot, C., W"oll, C.: "Comment on "imaging of the hydrogen subsurface site in rutile $\mathrm{TiO}_{2}$ ” Physical review letters 104(11), 119603 (2010) 
[48] Panayotov, D.A., Yates Jr, J.T.: n-type doping of $\mathrm{TiO}_{2}$ with atomic hydrogen-observation of the production of conduction band electrons by infrared spectroscopy. Chemical physics letters 436(1-3), 204-208 (2007)

[49] Deskins, N.A., Rousseau, R., Dupuis, M.: Localized electronic states from surface hydroxyls and polarons in $\mathrm{TiO}_{2}(110)$. The Journal of Physical Chemistry C 113(33), 14583-14586 (2009)

[50] Di Valentin, C., Pacchioni, G., Selloni, A.: Electronic structure of defect states in hydroxylated and reduced rutile $\mathrm{TiO}_{2}$ (110) surfaces. Physical review letters 97(16), 166803 (2006)

[51] Krüger, P., Jupille, J., Bourgeois, S., Domenichini, B., Verdini, A., Floreano, L., Morgante, A.: Intrinsic nature of the excess electron distribution at the $\mathrm{TiO}_{2}$ (110) surface. Physical review letters 108(12), 126803 (2012) 